\title{
Vacuum Stability, Perturbativity, EWPD and Higgs-to-diphoton rate in Type II Seesaw Models
}

\author{
Eung Jin Chun, Hyun Min Lee, and Pankaj Sharma \\ Korea Institute for Advanced Study \\ Heogiro 87, Dongdaemun-gu, Seoul 130-722, Korea \\ E-mail: ejchun@kias.re.kr, hyun.min.lee@kias.re.kr, pankajs@kias.re.kr
}

\begin{abstract}
We study constraints from perturbativity and vacuum stability as well as the EWPD in the type II seesaw model. As a result, we can put stringent limits on the Higgs triplet couplings depending on the cut-off scale. The EWPD tightly constrain the Higgs triplet mass splitting to be smaller than $40 \mathrm{GeV}$. Analyzing the Higgs-to-diphoton rate in the allowed parameter region, we show a possible enhancement by up to $100 \%$ and $50 \%$ for the cut-off scale of $100 \mathrm{TeV}$ and $10^{19} \mathrm{GeV}$, respectively, if the doubly charged Higgs boson mass is as low as $100 \mathrm{GeV}$.
\end{abstract}




\section{Contents}

1 Introduction 1

2 Higgs couplings in type II seesaw 3

3 Vacuum stability and perturbativity 4

4 Constraints from EWPD 6

$\begin{array}{lll}5 & \text { Higgs triplet contribution to } h \rightarrow \gamma \gamma & 7\end{array}$

$\begin{array}{lll}6 & \text { Results and summary } & 9\end{array}$

\section{Introduction}

The discovery of the Higgs boson at around $125 \mathrm{GeV}$ [1] opened a new era toward the Higgs precision test. It is essential for the LHC and future experiments to determine how precisely the Higgs candidate follows the very prediction of the Standard Model (SM), as new physics might enter here to modify the SM Higgs property in various ways.

One of the motivations for new physics beyond the SM comes from the smallness of neutrino masses whose origin can be attributed to a new particle coupling to the lepton doublets of the SM. In this paper, we consider the type II seesaw mechanism which introduces a Higgs triplet whose vacuum expectation value (VEV) generates the neutrino masses and mixing [2]. The Higgs sector of the type II seesaw contains four more bosons, $H^{++}, H^{+}$and $H^{0} / A^{0}$, in addition to the SM Higgs boson, $h$. While the standard Higgs doublet generates the quark and charged lepton masses, the Higgs triplet couples only to the lepton doublets generating the neutrino masses. This mechanism leads to a peculiar prediction of a same-sign dilepton resonance, $\mathrm{H}^{++} \rightarrow l_{\alpha}^{+} l_{\beta}^{+}$, which is being searched at the LHC [3]. As the Higgs triplet Yukawa matrix is proportional to the neutrino mass matrix, the observation of the flavor structure of the same-sign dilepton final states allows us to determine the neutrino mass pattern at colliders [4].

Other interesting features of the type II seesaw come from the Higgs boson sector. The Higgs triplet couplings can change drastically the stability of the SM electroweak vacuum [5] so they are quite constrained. Furthermore, in the limit of tiny lepton Yukawa couplings of the Higgs triplet, the triplet self couplings are also constrained by perturbativity as they tend to blow up rapidly. Considering the perturbativity and absolute vacuum stability conditions up to the Planck scale, we will see that perturbativity keeps a triplet self coupling, denoted by $\lambda_{2}$, smaller than 0.25 and then vacuum stability requires all the other 
couplings to be smaller than 0.5 . If a lower instability scale is taken, such a stringent limit can of course be relaxed, but not too much.

Another important constraint can be deduced from the electroweak precision data (EWPD) [6]. Note that one of the couplings between the Higgs triplet and doublet, denoted by $\lambda_{5}$, induces mass splitting $\Delta M$ among the triplet components [4]. The EWPD turn out to put a strong limit of $|\Delta M| \lesssim 40 \mathrm{GeV}$ allowing only a narrow range of $\lambda_{5}$ depending on the Higgs triplet mass when the triplet VEV is taken to be tiny enough so that its tree-level contribution to $\Delta \rho$ is neglected.

As noted in [7-9], the SM Higgs boson decay $h \rightarrow \gamma \gamma$ can be significantly modified through one-loop diagrams involving the charged Higgs bosons, in particular, $\mathrm{H}^{++}$, if quartic couplings mixing with the SM Higgs are large and the triplet mass is small. Recently it is of a particular interest to look for plausible models accommodating the enhanced Higgs-to-diphoton rate that appeared in the current LHC data [10]. Whether or not such a deviation is confirmed by a further LHC search, the precise measurement of the diphoton rate will place an important restriction on the type II seesaw model. In our analysis, we show how much the $h \rightarrow \gamma \gamma$ rate can deviate from the SM prediction after restricting ourselves to the model parameter space allowed by the perturbativity and vacuum stability conditions as well as the EWPD constraint, which has not been considered properly in the previous studies [7-9]. As expected, the result strongly depends on the assumed instability scale as well as the Higgs triplet mass. For our analysis, we will take the instability scale at $100 \mathrm{TeV}, 10^{10} \mathrm{GeV}$ and $10^{19} \mathrm{GeV}$. The doubly charged Higgs boson mass is taken to be as low as $100 \mathrm{GeV}$ although it can be even smaller depending on the assumed decay channels of the triplet components.

All the collider searches for the doubly charged Higgs boson so far look for the clean signal of $H^{++} \rightarrow l_{\alpha}^{+} l_{\beta}^{+}$. The previous results from LEP [11] and Tevatron [12] put lower limits on the charged Higgs boson mass at around $100 \mathrm{GeV}$ assuming the $100 \%$ branching fraction for the $\mathrm{H}^{++}$decay to some specific lepton flavours. The current CMS analysis includes more realistic dilepton decay channels reflecting the allowed neutrino mass pattern and puts the lower limit of $333 \mathrm{GeV}-408 \mathrm{GeV}$ depending on the chosen benchmark points and decay scenarios [3]. But this limit is applicable when the same-sign dilepton branching ratio is again $100 \%$. In fact, the doubly charged Higgs can have three types of decay channels: $H^{++} \rightarrow l_{\alpha}^{+} l_{\beta}^{+}, W^{+} W^{+}$and $H^{+} W^{+}$, one of which can dominate depending on the model parameters [13]. If one considers the triplet VEV larger than about $10^{-4} \mathrm{GeV}$, $H^{++}$can decay dominantly to $W^{+} W^{+}$reducing the dilepton branching ratio and thus the lower limit on the doubly charged Higgs boson mass. It may be worthwhile to make more serious studies to search for the $H^{++} \rightarrow W^{+} W^{+}$signal in the upcoming $14 \mathrm{TeV}$ LHC $[14,15]$. The worst scenario for the doubly charged Higgs search would be when there is a sizable mass gap among the triplet components and the doubly charged state is the heaviest (for $\lambda_{5}<0$ as we will see). In this case, the produced doubly charged Higgs boson follows the decay chain: $H^{++} \rightarrow H^{+} W^{(*)} \rightarrow H^{0} / A^{0} W^{(*)} W^{(*)} \rightarrow \nu \nu W^{(*)} W^{(*)}$ and thus the triplet can be completely missed.

This paper is organized as follows. After introducing the type II seesaw model with the 
model parameters and notations for our analysis in Section 2, we will find constraints on the Higgs couplings by applying the conditions for the perturbativity and vacuum stability, and the renormalization group equations at one-loop in Section 3. Then, additional restrictions from the EWPD will be obtained in Section 4. We will calculate the modified Higgs-todiphoton rate due to the $\mathrm{H}^{++}$and $\mathrm{H}^{+}$contribution depending on the allowed ranges of the Higgs triplet couplings in Section 5. The results of our combined analysis will be presented and conclusions will be drawn in Section 6 .

\section{Higgs couplings in type II seesaw}

When the Higgs sector of the Standard Model is extended with a $Y=2 S U(2)_{L}$ scalar triplet $\Delta$ in addition to a SM-Higgs doublet $\Phi$, the gauge-invariant Lagrangian is written as

$$
\mathcal{L}=\left(D_{\mu} \Phi\right)^{\dagger}\left(D^{\mu} \Phi\right)+\operatorname{Tr}\left(D_{\mu} \Delta\right)^{\dagger}\left(D^{\mu} \Delta\right)-\mathcal{L}_{Y}-V(\Phi, \Delta)
$$

where the leptonic part of the Lagrangian required to generate neutrino masses is

$$
\mathcal{L}_{Y}=f_{\alpha \beta} L_{\alpha}^{T} C i \tau_{2} \Delta L_{\beta}+\text { H.c. }
$$

and the scalar potential is

$$
\begin{aligned}
V(\Phi, \Delta) & =m^{2} \Phi^{\dagger} \Phi+\lambda_{1}\left(\Phi^{\dagger} \Phi\right)^{2}+M^{2} \operatorname{Tr}\left(\Delta^{\dagger} \Delta\right) \\
& +\lambda_{2}\left[\operatorname{Tr}\left(\Delta^{\dagger} \Delta\right)\right]^{2}+2 \lambda_{3} \operatorname{Det}\left(\Delta^{\dagger} \Delta\right)+\lambda_{4}\left(\Phi^{\dagger} \Phi\right) \operatorname{Tr}\left(\Delta^{\dagger} \Delta\right) \\
& +\lambda_{5}\left(\Phi^{\dagger} \tau_{i} \Phi\right) \operatorname{Tr}\left(\Delta^{\dagger} \tau_{i} \Delta\right)+\left[\frac{1}{\sqrt{2}} \mu\left(\Phi^{T} i \tau_{2} \Delta^{\dagger} \Phi\right)+\text { H.c. }\right] .
\end{aligned}
$$

Here used is the $2 \times 2$ matrix representation of $\Delta$ :

$$
\Delta=\left(\begin{array}{cc}
\Delta^{+} / \sqrt{2} & \Delta^{++} \\
\Delta^{0} & -\Delta^{+} / \sqrt{2}
\end{array}\right) .
$$

Upon the electroweak symmetry breaking with $\left\langle\Phi^{0}\right\rangle=v_{0} / \sqrt{2}$, the $\mu$ term in Eq. (2.2) gives rise to the vacuum expectation value of the triplet $\left\langle\Delta^{0}\right\rangle=v_{\Delta} / \sqrt{2}$ where $v_{\Delta} \approx \mu v_{0}^{2} / \sqrt{2} M^{2}$. We will assume $\mu$ is real positive without loss of generality. From the leptonic Yukawa coupling (2.1), one can get the neutrino mass matrix

$$
M_{\alpha \beta}^{\nu}=f_{\alpha \beta} \xi v_{0},
$$

where $\xi \equiv v_{\Delta} / v_{0}$. The observed neutrino mass of order $0.1 \mathrm{eV}$ requires $\left|f_{\alpha \beta} \xi\right| \sim 10^{-12}$. Considering this relation, we will assume $\left|f_{\alpha \beta}\right| \ll 1$ and $|\xi| \ll 1$ throughout this work. Let us remind that the measurement of $\rho \equiv M_{W}^{2} /\left(M_{Z}^{2} c_{W}^{2}\right) \approx 1$ puts the bound $\xi \lesssim 10^{-2}$. Since we further take the region of $|\xi| \ll 10^{-2}$ in our analysis, some of the effects with a largest possible value of $\xi[8,16]$ can be safely neglected.

After the electroweak symmetry breaking, there are five physical massive bosons denoted by $H^{ \pm \pm}, H^{ \pm}, H^{0}, A^{0}, h^{0}$. Under the condition of $|\xi| \ll 1$, the first five states 
are mainly from the triplet scalar and the last from the doublet scalar. For the neutral pseudoscalar and charged scalar parts,

$$
\begin{aligned}
\phi_{I}^{0} & =G^{0}-2 \xi A^{0}, & \phi^{+} & =G^{+}+\sqrt{2} \xi H^{+} \\
\Delta_{I}^{0} & =A^{0}+2 \xi G^{0}, & \Delta^{+} & =H^{+}-\sqrt{2} \xi G^{+}
\end{aligned}
$$

where $G^{0}$ and $G^{+}$are the Goldstone modes, and for the neutral scalar part,

$$
\begin{aligned}
\phi_{R}^{0} & =h^{0}-a \xi H^{0}, \\
\Delta_{R}^{0} & =H^{0}+a \xi h^{0}
\end{aligned}
$$

where $a=2+\left(4 \lambda_{1}-\lambda_{4}-\lambda_{5}\right) v_{0}^{2} /\left(M_{H^{0}}^{2}-M_{h^{0}}^{2}\right)$. The masses of the Higgs bosons essentially from the triplet are

$$
\begin{aligned}
M_{H^{ \pm \pm}}^{2} & =M^{2}+\frac{\lambda_{4}-\lambda_{5}}{2} v_{0}^{2} \\
M_{H^{ \pm}}^{2} & =M_{H^{ \pm \pm}}^{2}+\frac{\lambda_{5}}{2} v_{0}^{2} \\
M_{H^{0}, A^{0}}^{2} & =M_{H^{ \pm \pm}}^{2}+\lambda_{5} v_{0}^{2},
\end{aligned}
$$

neglecting small contributions from $v_{\Delta}$. The mass of $h^{0}$ is given by $m_{h^{0}}^{2}=2 \lambda_{1} v_{0}^{2}$ as usual.

Eq. (2.7) tells us that the mass splitting, $\Delta M \equiv M_{H^{ \pm}}-M_{H^{ \pm \pm}}$, is driven by the coupling $\lambda_{5}$ which affects also the EWPD and the Higgs-to-diphoton rate. Recall that depending upon the sign of the coupling $\lambda_{5}$, there are two mass hierarchies among the triplet components: $M_{H^{ \pm \pm}}>M_{H^{ \pm}}>M_{H^{0}, A^{0}}$ for $\lambda_{5}<0$; or $M_{H^{ \pm \pm}}<M_{H^{ \pm}}<M_{H^{0}, A^{0}}$ for $\lambda_{5}>0$ [4]. The charged Higgs boson as light as $100 \mathrm{GeV}\left(M_{H^{ \pm \pm}}\right.$or $\left.M_{H^{ \pm}}=100 \mathrm{GeV}\right)$ can evade the CMS search if the decay channels of $H^{ \pm \pm} \rightarrow H^{ \pm} W^{*}$ and $H^{ \pm} \rightarrow H^{0} / A^{0} W^{*}$ are the dominant modes allowed by a sizable $\lambda_{5}$ in the first case, or if $H^{ \pm \pm}$decays dominantly to $W^{ \pm} W^{ \pm}$with $|\xi| \gg\left|f_{i j}\right|$ in the second case.

\section{Vacuum stability and perturbativity}

The scalar potential $(2.2)$ contains seven free parameters: $\lambda_{i}(i=1 \ldots 5), v_{\Delta}$ and $M_{H^{++}}$. Rather stringent constraints on these parameters can be readily obtained by the theoretical requirements of perturbativity and vacuum stability. A detailed study of the scalar potential has been performed in [17]. The vacuum stability conditions on the scalar couplings $\lambda_{i}$ are as follows:

$$
\begin{aligned}
& \lambda_{1}>0, \quad \lambda_{2}>0, \quad \lambda_{2}+\frac{1}{2} \lambda_{3}>0 \\
& \lambda_{4} \pm \lambda_{5}+2 \sqrt{\lambda_{1} \lambda_{2}}>0, \quad \lambda_{4} \pm \lambda_{5}+2 \sqrt{\lambda_{1}\left(\lambda_{2}+\frac{1}{2} \lambda_{3}\right)}>0 .
\end{aligned}
$$

Apart from these conditions, we will put the perturbativity conditions: $\left|\lambda_{i}\right| \leq \sqrt{4 \pi}$.

We will take the absolute stability condition ${ }^{1}$ that all these constraints must remain true up to the scale where the theory is supposed to be valid. Henceforth, we study the

\footnotetext{
${ }^{1}$ Imposing metastability [19] instead of absolute stability would lead to a wider parameter space but we don't consider this possibility in our work.
} 
renormalization group (RG) evolution of these scalar couplings $\left(\lambda_{i}\right.$ 's), EW-gauge couplings $g_{2}, g^{\prime}$, strong coupling $g_{3}$ and top-Yukawa coupling $y_{t}$ up to the cut-off scale at the one-loop level. The RG evolution of the type II seesaw model has been studied in [18]. The one-loop RG equations relevant for our analysis are as below:

$$
\begin{aligned}
16 \pi^{2} \frac{d g^{\prime}}{d t} & =\frac{47}{6} g^{\prime 3}, \quad 16 \pi^{2} \frac{d g_{2}}{d t}=-\frac{5}{2} g_{2}^{3}, \quad 16 \pi^{2} \frac{d g_{3}}{d t}=-7 g_{3}^{3} \\
16 \pi^{2} \frac{d y_{t}}{d t} & =y_{t}\left(\frac{9}{2} y_{t}^{2}-\frac{17}{12} g^{\prime 2}-\frac{9}{4} g_{2}^{2}-8 g_{3}^{2}\right) \\
16 \pi^{2} \frac{d \lambda_{1}}{d t} & =24 \lambda_{1}^{2}+\lambda_{1}\left(-9 g_{2}^{2}-3 g^{\prime 2}+12 y_{t}^{2}\right)+\frac{3}{4} g_{2}^{4}+\frac{3}{8}\left(g^{\prime 2}+g_{2}^{2}\right)^{2}-6 y_{t}^{4}+3 \lambda_{4}^{2}+2 \lambda_{5}^{2} \\
16 \pi^{2} \frac{d \lambda_{2}}{d t} & =\lambda_{2}\left(-12 g^{\prime 2}-24 g_{2}^{2}\right)+6 g^{\prime 4}+9 g_{2}^{4}+12 g^{\prime 2} g_{2}^{2}+28 \lambda_{2}^{2}+8 \lambda_{2} \lambda_{3}+4 \lambda_{3}^{2}+2 \lambda_{4}^{2}+2 \lambda_{5}^{2} \\
16 \pi^{2} \frac{d \lambda_{3}}{d t} & =\lambda_{3}\left(-12 g^{\prime 2}-24 g_{2}^{2}\right)+6 g_{2}^{4}-24 g^{\prime 2} g_{2}^{2}+6 \lambda_{3}^{2}+24 \lambda_{2} \lambda_{3}-4 \lambda_{5}^{2} \\
16 \pi^{2} \frac{d \lambda_{4}}{d t} & =\lambda_{4}\left(-\frac{15}{2} g^{\prime 2}-\frac{33}{2} g_{2}^{2}\right)+\frac{9}{5} g^{\prime 4}+6 g_{2}^{4}+\lambda_{4}\left(12 \lambda_{1}+16 \lambda_{2}+4 \lambda_{3}+4 \lambda_{4}+6 y_{t}^{2}\right)+8 \lambda_{5}^{2} \\
16 \pi^{2} \frac{d \lambda_{5}}{d t} & =\lambda_{4}\left(-\frac{15}{2} g^{\prime 2}-\frac{33}{2} g_{2}^{2}\right)+6 g^{\prime 2} g_{2}^{2}+\lambda_{5}\left(4 \lambda_{1}+4 \lambda_{2}-4 \lambda_{3}+8 \lambda_{4}+6 y_{t}^{2}\right),
\end{aligned}
$$

where $t \equiv \ln \left(\mu / M_{t}\right)$ and the contributions from the neutrino Yukawa couplings, $f_{\alpha \beta}$, are neglected.
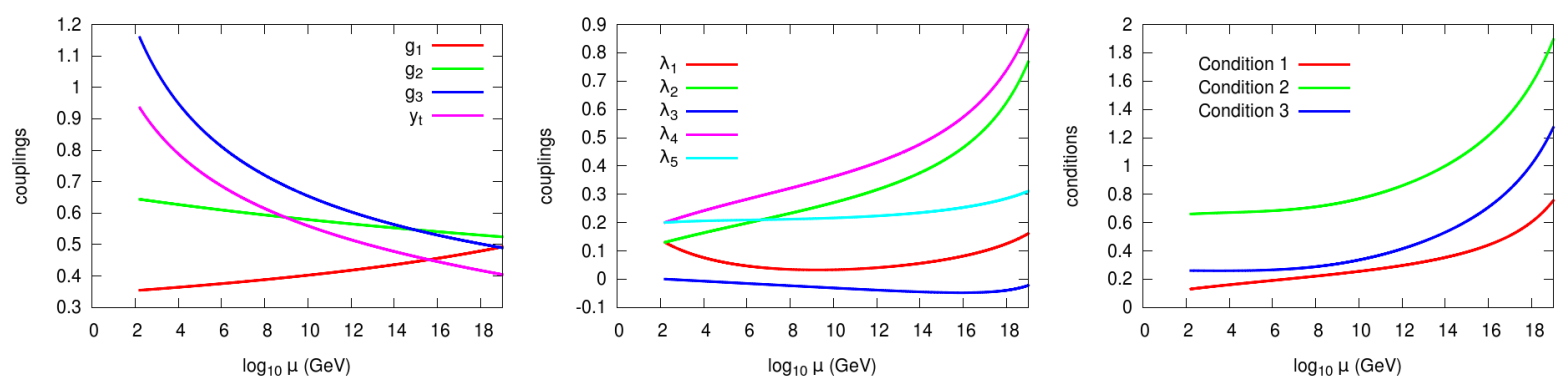

Figure 1. RG evolution of couplings and vacuum stability conditions.

In Fig. 1, we show an example of the RG running of the couplings which maintain the perturbativity and vacuum stability up to the Planck scale. In the rightmost panel, the three vacuum stability conditions; (1) $\lambda_{2}+\frac{1}{2} \lambda_{3}>0$, (2) $\lambda_{4}-\lambda_{5}+2 \sqrt{\lambda_{1} \lambda_{2}}>0$, and (3) $\lambda_{4}-\lambda_{5}+2 \sqrt{\lambda_{1}\left(\lambda_{2}+\frac{1}{2} \lambda_{3}\right)}>0$ are presented. Note that the Higgs doublet selfcoupling $\lambda_{1}$ decreases initially due to the top Yukawa coupling as in the SM, but it turns around to increase at a certain point with the aid of other increasing couplings. For our numerical analysis, we use $M_{t}=173 \mathrm{GeV}, m_{t}\left(M_{t}\right)=164 \mathrm{GeV}, m_{h}=125 \mathrm{GeV}$ and thus $\lambda_{1}\left(M_{t}\right)=m_{h}^{2} / 2 v_{0}^{2}=0.129$ and $y_{t}\left(M_{t}\right)=\sqrt{2} m_{t} / v_{0}=0.938$. 


\section{Constraints from EWPD}

In this section, we study the contributions of the Higgs triplet to the EWPD observables, also known as the oblique parameters. In [20], the contribution of a scalar multiplet of arbitrary weak isospin and weak hypercharge to the $S, T$ and $U$ parameters has been calculated. We present here the expressions for the specific case of the Higgs triplet model:

$$
\begin{array}{r}
S=-\frac{1}{3 \pi} \ln \frac{m_{+1}^{2}}{m_{-1}^{2}}-\frac{2}{\pi} \sum_{T_{3}=-1}^{+1}\left(T_{3}-Q s_{W}^{2}\right)^{2} \xi\left(\frac{m_{T_{3}}^{2}}{m_{Z}^{2}}, \frac{m_{T_{3}}^{2}}{m_{Z}^{2}}\right) \\
T=\frac{1}{16 \pi c_{W}^{2} s_{W}^{2}} \sum_{T_{3}=-1}^{+1}\left(2-T_{3}\left(T_{3}-1\right)\right) \eta\left(\frac{m_{T_{3}}^{2}}{m_{Z}^{2}}, \frac{m_{T_{3}-1}^{2}}{m_{Z}^{2}}\right) \\
\begin{aligned}
U=\frac{1}{6 \pi} \ln \frac{m_{0}^{4}}{m_{+1}^{2} m_{-1}^{2}}+\frac{1}{\pi} \sum_{T_{3}=-1}^{+1}\left[2\left(T_{3}-Q s_{W}^{2}\right)^{2} \xi\left(\frac{m_{T_{3}}^{2}}{m_{Z}^{2}}, \frac{m_{T_{3}}^{2}}{m_{Z}^{2}}\right)\right. \\
\left.\quad-\left(2-T_{3}\left(T_{3}-1\right)\right) \xi\left(\frac{m_{T_{3}}^{2}}{m_{W}^{2}}, \frac{m_{T_{3}}^{2}}{m_{W}^{2}}\right)\right]
\end{aligned}
\end{array}
$$

where $m_{+1,0,-1}=M_{H^{++}, H^{+}, H^{0}}$ and the functions $\xi(x, y)$ and $\eta(x, y)$ are defined by

$$
\begin{aligned}
\xi(x, y)= & \frac{4}{9}-\frac{5}{12}(x+y)+\frac{1}{6}(x-y)^{2} \\
& +\frac{1}{4}\left[x^{2}-y^{2}-\frac{1}{3}(x-y)^{3}-\frac{x^{2}+y^{2}}{x-y}\right] \ln \frac{x}{y}-\frac{1}{12} d(x, y) f(x, y) \\
d(x, y)= & -1+2(x+y)-(x-y)^{2} \\
f(x, y)= & \left\{\begin{array}{l}
-2 \sqrt{d(x, y)}\left[\arctan \frac{x-y+1}{\sqrt{d(x, y)}}-\arctan \frac{x-y-1}{\sqrt{d(x, y)}}\right] \quad \text { for } d(x, y)>0 \\
\sqrt{-d(x, y)} \ln \left[\frac{x+y-1+\sqrt{-d(x, y)}}{x+y-1-\sqrt{-d(x, y)}}\right] \text { for } d(x, y) \leq 0
\end{array}\right. \\
\eta(x, y)= & x+y-\frac{2 x y}{x-y} \ln \frac{x}{y}
\end{aligned}
$$

Adopting the most recent fit results for the allowed regions of the $S, T$ and $U$ presented in [21], we use the following values for the SM fit of the oblique parameters:

$$
\begin{array}{ll}
S_{\text {best fit }}=0.03, & \sigma_{S}=0.10, \\
T_{\text {best fit }}=0.05, & \sigma_{T}=0.12, \\
U_{\text {best fit }}=0.03, & \sigma_{U}=0.10,
\end{array}
$$

As the $S, T$ and $U$ are not independent quantities, there is a correlation among these quantities. The correlation coefficients are given by

$$
\rho_{S T}=0.89, \quad \rho_{S U}=-0.54, \quad \rho_{T U}=-0.83
$$


The contour allowed by the EWPD at a given confidence level $C L$ is then determined by

$$
\left(\begin{array}{c}
S-S_{\text {best fit }} \\
T-T_{\text {best fit }} \\
U-U_{\text {best fit }}
\end{array}\right)^{T}\left(\begin{array}{ccc}
\sigma_{S} \sigma_{S} & \sigma_{S} \sigma_{T} \rho_{S T} & \sigma_{S} \sigma_{U} \rho_{S U} \\
\sigma_{S} \sigma_{T} \rho_{S T} & \sigma_{T} \sigma_{T} & \sigma_{T} \sigma_{U} \rho_{T U} \\
\sigma_{U} \sigma_{S} \rho_{U S} & \sigma_{U} \sigma_{T} \rho_{T U} & \sigma_{U} \sigma_{U}
\end{array}\right)^{-1}\left(\begin{array}{c}
S-S_{\text {best fit }} \\
T-T_{\text {best fit }} \\
U-U_{\text {best fit }}
\end{array}\right)=-2 \ln (1-C L) \text {. }
$$

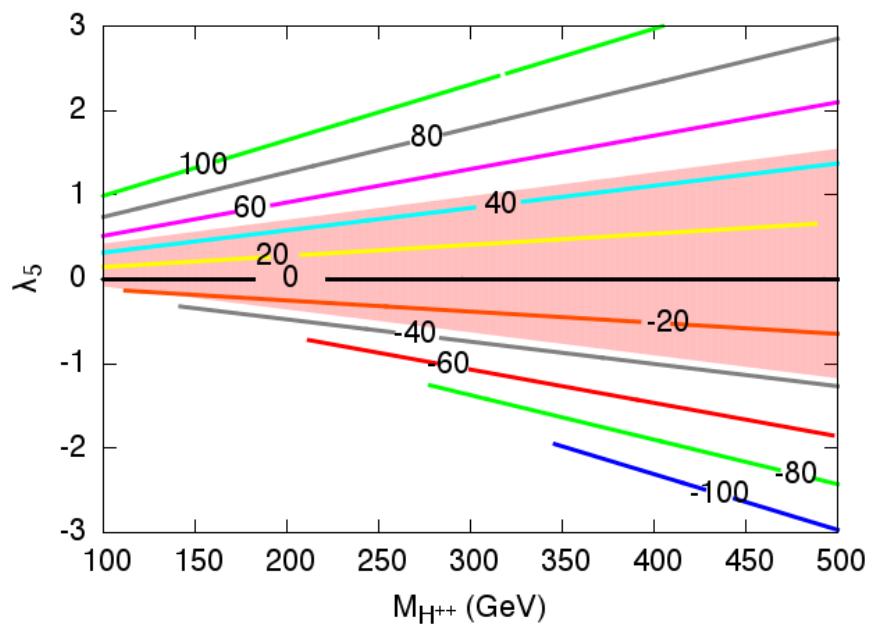

Figure 2. Allowed parameter space in the $M_{H^{++}} \lambda_{5}$ plane. The contours represent the allowed values of mass splitting, $\Delta M \equiv M_{H^{+}}-M_{H^{++}}$, in the unit of $\mathrm{GeV}$. The shaded band denotes the 99\% CL region satisfying the EWPD constraint.

In Fig. 2, we show the allowed parameter space in the $M_{H^{++}}-\lambda_{5}$ plane consistent with the EWPD. The shaded region shows the EWPD constraint at 99\% CL. The contour lines show the mass splitting, $\Delta M \equiv M_{H^{+}}-M_{H^{++}}$, from which one can see that the mass splitting is tightly constrained to be within $|\Delta M| \lesssim 40 \mathrm{GeV}$ independently of the doubly charged Higgs mass.

Note that this conclusion can be changed if a relatively large triple VEV, $v_{\Delta} \gtrsim 1 \mathrm{GeV}$ $(\xi \sim 0.01)$, is assumed [8] in which case a sizable tree-level $\delta \rho$ contribution coming from the triplet VEV can be cancelled out by loop contributions with a large mass splitting among the triplet components to satisfy the EWPD constraints.

\section{$5 \quad$ Higgs triplet contribution to $h \rightarrow \gamma \gamma$}

Having studied the consistency conditions on the model parameters, we now analyze their impact on the Higgs boson decay to two photons. In the type II seesaw model, the Higgsto-diphoton decay rate gets a sizable contribution from the charged Higgs bosons, $\mathrm{H}^{++}$and $H^{+}$, which can lead to a constructive or destructive interference with the SM contribution from the top quark and weak gauge boson. Summing up all the contributions, one gets the 
following Higgs-to-diphoton rate [22]:

$$
\begin{aligned}
\Gamma(h \rightarrow \gamma \gamma)= & \frac{G_{F} \alpha^{2} m_{h}^{3}}{128 \sqrt{2} \pi^{3}} \mid \sum_{f} N_{c} Q_{f}^{2} g_{f f}^{h} A_{1 / 2}^{h}\left(x_{f}\right)+g_{W W}^{h} A_{1}^{h}\left(x_{W}\right) \\
& +g_{H^{+} H^{-}}^{h} A_{0}^{h}\left(x_{H^{+}}\right)+\left.4 g_{H^{++} H^{--}}^{h} A_{0}^{h}\left(x_{H^{++}}\right)\right|^{2}
\end{aligned}
$$

where $x_{i}=m_{h}^{2} / 4 m_{i}^{2}$ and the functions are

$$
\begin{aligned}
A_{1 / 2}^{h}(x) & =2 x^{-2}[x+(x-1) f(x)] \\
A_{1}^{h}(x) & =-x^{-2}\left[2 x^{2}+3 x+3(2 x-1) f(x)\right] \\
A_{0}^{h}(x) & =-x^{-2}[x-f(x)] \\
\text { where } & f(x)=\left\{\begin{array}{l}
\arcsin ^{2} \sqrt{x} \quad \text { for } \quad x \leq 1 \\
-\frac{1}{4}\left[\ln \frac{1+\sqrt{1-x^{-1}}}{1-\sqrt{1-x^{-1}}}-i \pi\right]^{2}
\end{array} \text { for } x>1\right.
\end{aligned}
$$

The Higgs couplings are $g_{f f}^{h}=1$ for the top and $g_{W W}^{h}=1$, whereas the Higgs triplet couplings are

$$
g_{H^{+} H^{+}}^{h}=\frac{\lambda_{4}}{2} \frac{v_{0}^{2}}{M_{H^{+}}^{2}}, \quad \text { and } \quad g_{H^{++} H^{++}}^{h}=\frac{\lambda_{4}-\lambda_{5}}{2} \frac{v_{0}^{2}}{M_{H^{++}}^{2}} .
$$

Since the SM contribution amounts to about -6.5 in the amplitude, negative values of $\lambda_{4}$ and $\lambda_{4}-\lambda_{5}$ can make a constructive interference to enhance the diphoton rate. As we will see in the next section, however, the vacuum stability condition strongly disfavors negative $\lambda_{4}$ and $\lambda_{4}-\lambda_{5}$ and allows more parameter region leading to a destructive interference to reduce the diphoton rate.
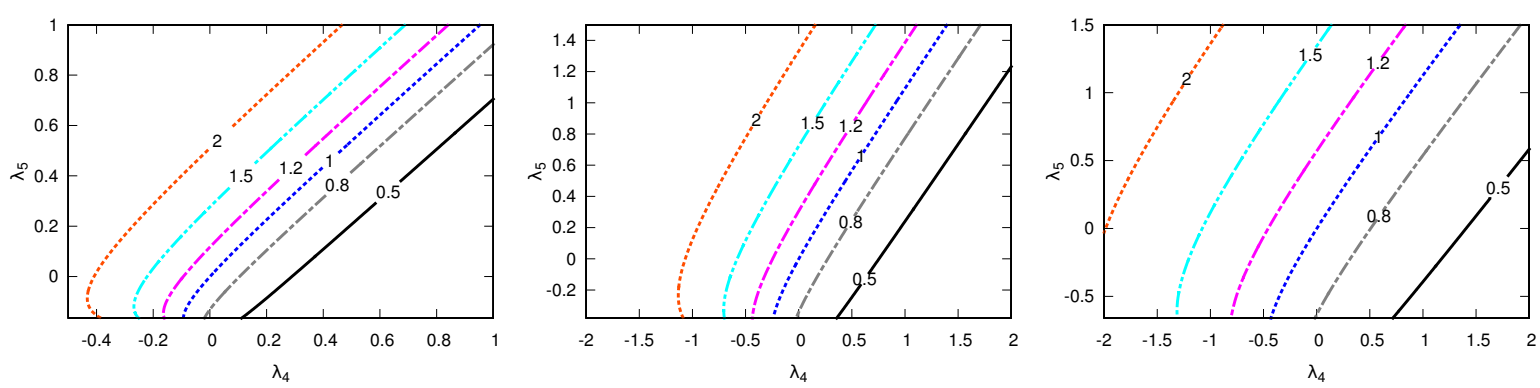

Figure 3. The $R_{\gamma \gamma}$ contours in the $\lambda_{4} \lambda_{5}$ plane for $M_{H^{++}}=100 \mathrm{GeV}$ (left), $150 \mathrm{GeV}$ (middle) and $200 \mathrm{GeV}$ (right).

Fig. 3 shows the contour lines of $R_{\gamma \gamma} \equiv \Gamma(h \rightarrow \gamma \gamma) /\left.\Gamma(h \rightarrow \gamma \gamma)\right|_{\mathrm{SM}}$ in the $\lambda_{4}-\lambda_{5}$ plane for the doubly charged Higgs masses, $M_{H^{++}}=100,150$, and $200 \mathrm{GeV}$. In the region with $\lambda_{5}<0$, the singly charged Higgs is lighter than the doubly charged Higgs and its constructive contribution becomes more sizable so that the contour lines start to bend 
for a certain value of $\lambda_{5}$. The contour lines are cut at the $\lambda_{5}$ values beyond which the mass-squared values of the neutral components of triplet become negative. Note that for $M_{H^{++}}=100 \mathrm{GeV}, 150 \mathrm{GeV}$ and $200 \mathrm{GeV}$, the positivity of mass-squared values of the neutral Higgs requires $\lambda_{5} \geq-0.165,-0.38$ and -0.66 respectively. In the next section, the constraints derived in the previous two sections are combined and overlayed with the $R_{\gamma \gamma}$ contours. We will see that the EWPD constraint derived in Fig. 2 restricts $\lambda_{5}$ to a smaller region than in Fig. 3.

\section{$6 \quad$ Results and summary}

In this section we perform a numerical analysis to constrain the parameter space of the scalar couplings by considering the conditions of vacuum stability and perturbativity up to the scale where the theory is considered to be valid. We present our results for three instability scales: $100 \mathrm{TeV}, 10^{10} \mathrm{GeV}$ and $10^{19} \mathrm{GeV}$ in Fig. 4, 5 and 6, respectively. We further look for the allowed parameter space combining these with the EWPD and quantify the deviation of the ratio $R_{\gamma \gamma}$ from the SM value $R_{\gamma \gamma}^{S M}=1$. Figs. $4-6$ summarize our results in the $\lambda_{4}-\lambda_{5}$ plane with different values of $\lambda_{2}$ and $\lambda_{3}$ for the doubly charged Higgs mass, $M_{H^{++}}=100 \mathrm{GeV}$ (left), $150 \mathrm{GeV}$ (middle) and $200 \mathrm{GeV}$ (right). The contours represent the values of $R_{\gamma \gamma}$. The gray (purple) bands denote the $99 \%$ (95\% CL) region satisfying the EWPD constraints.

It is obvious from the Figs. 4, 5 and 6 that the small cut-off scale allows a large parameter space while the larger cut-off scale constrains it. The allowed range of $\lambda_{2}$ depends upon the range of $\lambda_{3}$. Disregarding such a correlation, the maximally allowed ranges of the couplings depending on the cut-off scale are found to be as follows:

\begin{tabular}{c|c|c|c} 
& $10^{5} \mathrm{GeV}$ & $10^{10} \mathrm{GeV}$ & $10^{19} \mathrm{GeV}$ \\
\hline$\lambda_{2}$ & $(0,1)$ & $(0,0.5)$ & $(0,0.25)$ \\
$\lambda_{3}$ & $(-2.0,2.4)$ & $(-1.0,1.25)$ & $(-0.55,0.62)$ \\
$\lambda_{4}$ & $(-0.5,1.7)$ & $(-0.1,0.9)$ & $(0,0.5)$ \\
$\lambda_{5}$ & $(-1.5,1.5)$ & $(-0.7,0.7)$ & $(-0.4,0.4)$
\end{tabular}

The EWPD requiring the triplet mass splitting $|\Delta M| \lesssim 40 \mathrm{GeV}$, allow the following ranges of $\lambda_{5}$

$$
\lambda_{5}=(-0.1,0.4), \quad(-0.2,0.6), \quad(-0.35,0.7)
$$

for $M_{H^{++}}=100,150$, and $200 \mathrm{GeV}$, respectively.

We infer from these figures that for negative $\lambda_{3}$, larger values of $\lambda_{2}$ are allowed while for positive $\lambda_{3}$, smaller values of $\lambda_{2}$ are preferred to satisfy vacuum stability conditions. We observe that a large $\lambda_{2}$ tends to squeeze the allowed parameter space in the $\lambda_{4}-\lambda_{5}$ plane. This is due to the fact that a large $\lambda_{2}$ violates perturbativity very quickly when we evolve the coupling with RG equations. We find that $\lambda_{3}=0$ allows for a larger parameter space compared to two extremal values of $\lambda_{3}$. As a result, the enhancement of $R_{\gamma \gamma}$ is feasible for relatively larger allowed parameter space. The shaded bands in figures denote the allowed region by the EWPD depending on the doubly charged Higgs boson mass. As is obvious, 

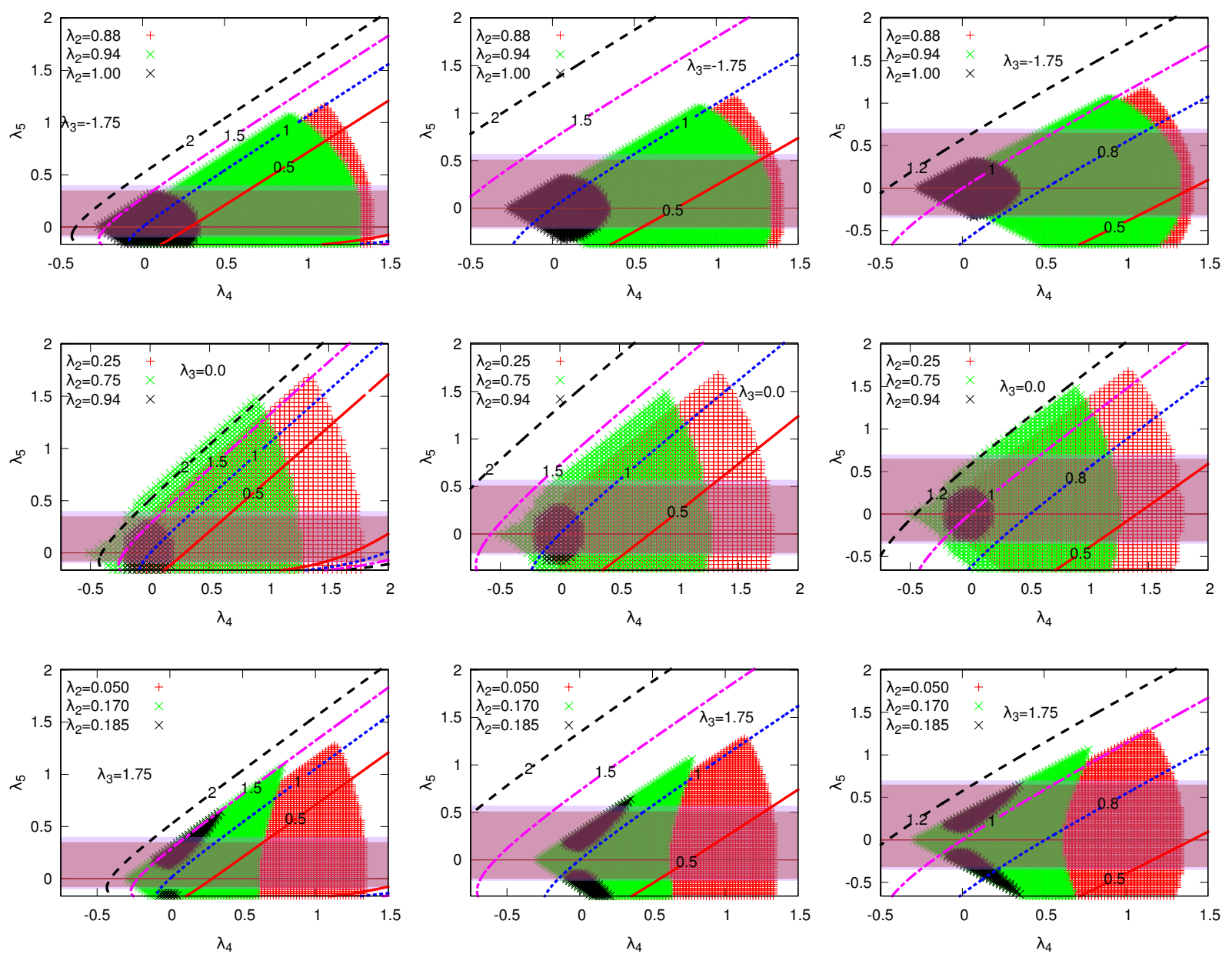

Figure 4. Allowed parameter space in the $\lambda_{4}-\lambda_{5}$ plane with different values of $\lambda_{2}$ and $\lambda_{3}$ for the doubly charged Higgs mass, $M_{H^{++}}=100 \mathrm{GeV}$ (left), $150 \mathrm{GeV}$ (middle) and $200 \mathrm{GeV}$ (right). The contours represent the values of $R_{\gamma \gamma}$. The gray (purple) bands denote the $99 \%$ (95\% CL) region satisfying the EWPD constraints. The cut-off scale is assumed to be $10^{5} \mathrm{GeV}$.

smaller and more positive ranges of $\lambda_{5}$ are allowed for smaller values of $M_{H^{++}}$. Although the allowed bands of $\lambda_{5}$ get smaller for smaller $M_{H^{++}}, R_{\gamma \gamma}$ can be more enhanced in these regions due to the sizable contribution from light charged Higgs bosons, in particular, near $\lambda_{4}=0$ favored by vacuum stability conditions. In the case of $M_{H^{++}}=100(200) \mathrm{GeV}$, one can get $R_{\gamma \gamma}$ as large as $2(1.2)$, or $1.5(1.1)$ for $\Lambda=10^{5}$, or $10^{10}$ and $10^{19} \mathrm{GeV}$. Of course, a larger parameter space opens up for a larger positive $\lambda_{4}$ for which a destructive interference occurs and thus $R_{\gamma \gamma}$ can be much smaller than 1 . Thus, broad ranges with positive $\lambda_{4}$ are strongly disfavored by the current LHC data.

To summarize, we studied the parameter space of the Higgs scalar potential of the type II seesaw model in the light of vacuum stability, perturbativity and EWPD constraints. Then we looked at the possible deviation in the Higgs-to-diphoton rate in the allowed parameter space. The allowed parameter space is found to be very restrictive and strongly 

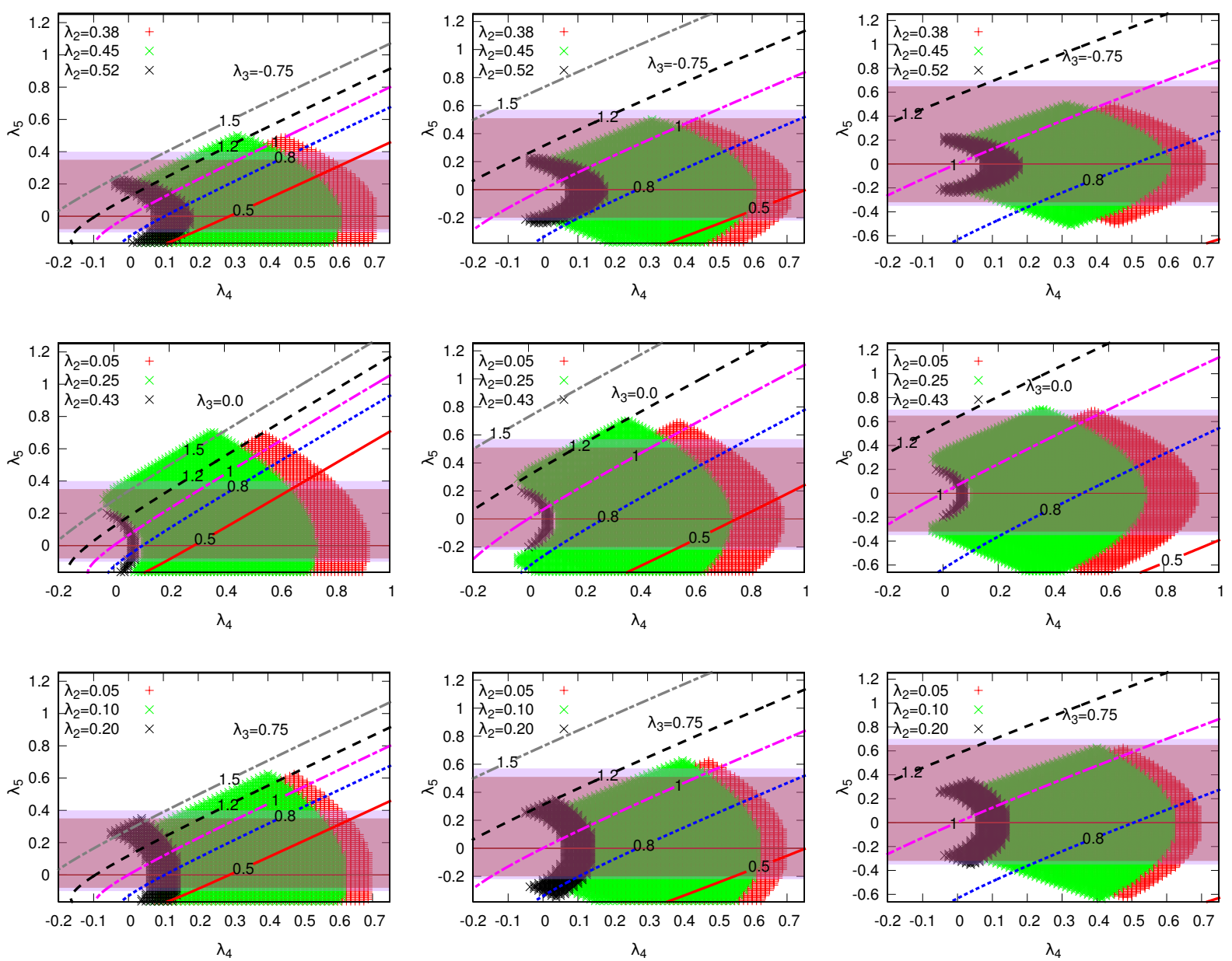

Figure 5. The same as Fig. 4, apart from that the cut-off scale is assumed to be $10^{10} \mathrm{GeV}$.

depend on the choice of the instability scale. Regardless of any choice of instability scale, $R_{\gamma \gamma}$ becomes smaller than 1 in a larger parameter space, but it can be enhanced by $50 \%$ $100 \%$ in some limited parameter region. If the deviation of the Higgs-to-diphoton rate turns out to be small with more data at the LHC, only a narrow band around $\lambda_{4} \approx \lambda_{5}$ will survive for low Higgs triplet mass.

\section{Acknowledgments}

EJC was supported by the National Research Foundation of Korea (NRF) grant funded by the Korea government (MEST) (No. 20120001177).

\section{References}

[1] G. Aad et al. [The ATLAS Collaboration], arXiv:1207.7214 [hep-ex]; S. Chatrchyan et al. [The CMS Collaboration], arXiv:1207.7235 [hep-ex]. 

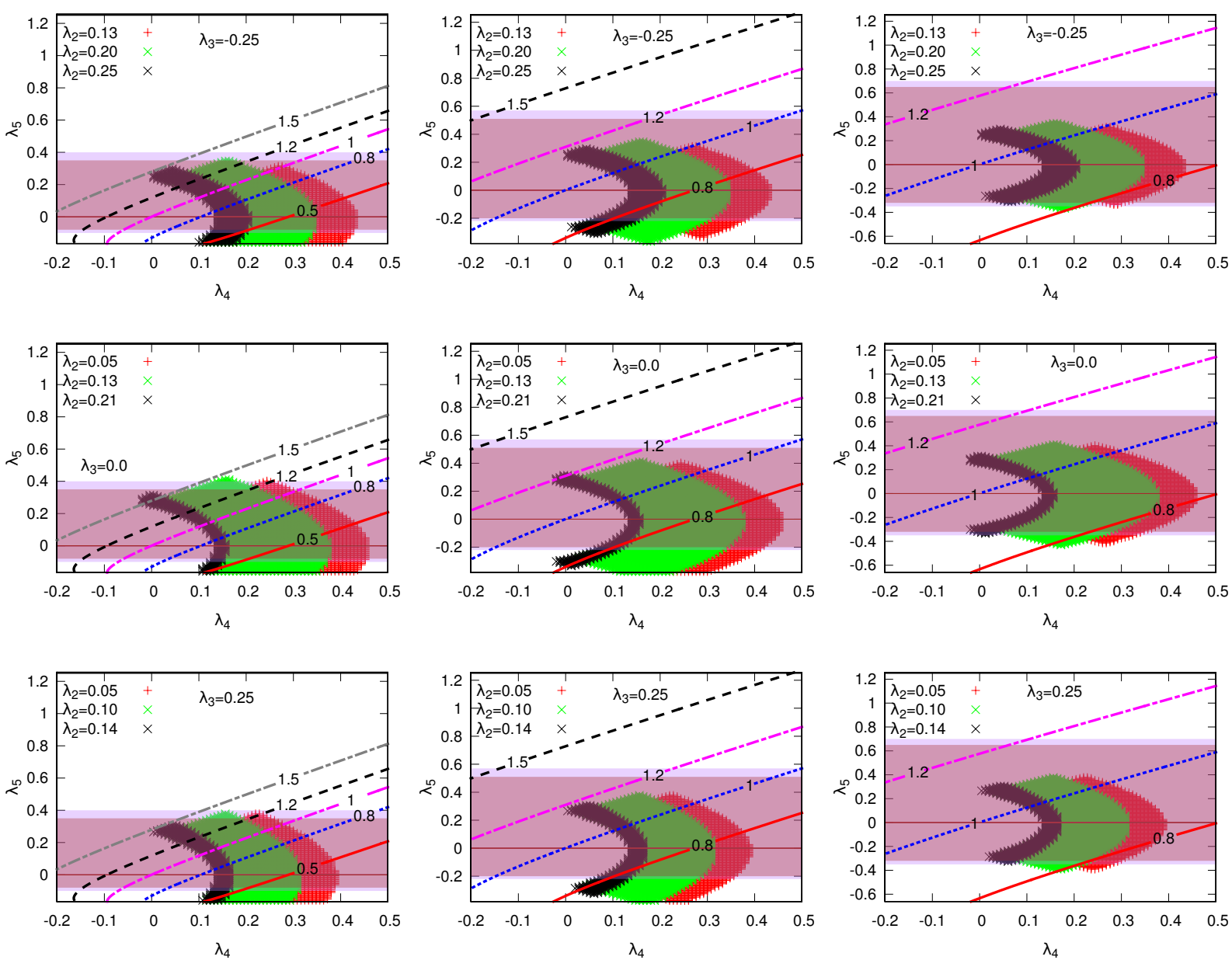

Figure 6. The same as Fig. 4, apart from that the cut-off scale is assumed to be $10^{19} \mathrm{GeV}$.

[2] M. Magg and C. Wetterich, Phys. Lett. B 94 (1980) 61; J. Schechter and J. W. F. Valle, Phys. Rev. D 22 (1980) 2227. T. P. Cheng and L. -F. Li, Phys. Rev. D 22 (1980) 2860; R. N. Mohapatra and G. Senjanovic, Phys. Rev. D 23 (1981) 165.

[3] S. Chatrchyan et al. [CMS Collaboration], arXiv:1207.2666 [hep-ex].

[4] E. J. Chun, K. Y. Lee and S. C. Park, Phys. Lett. B 566 (2003) 142 [hep-ph/0304069].

[5] N. Cabibbo, L. Maiani, G. Parisi and R. Petronzio, Nucl. Phys. B 158 (1979) 295; P. Q. Hung, Phys. Rev. Lett. 42 (1979) 873; M. Lindner, Z. Phys. C 31 (1986) 295; M. Lindner, M. Sher and H. W. Zaglauer, Phys. Lett. B 228 (1989) 139; M. Sher, Phys. Rept. 179 (1989) 273; J. Elias-Miro, J. R. Espinosa, G. F. Giudice, G. Isidori, A. Riotto and A. Strumia, Phys. Lett. B 709 (2012) 222 [arXiv:1112.3022 [hep-ph]]; G. Degrassi, S. Di Vita, J. Elias-Miro, J. R. Espinosa, G. F. Giudice, G. Isidori and A. Strumia, JHEP 1208 (2012) 098 [arXiv:1205.6497 [hep-ph]]. I. Masina, arXiv:1209.0393 [hep-ph].

[6] A. Melfo, M. Nemevsek, F. Nesti, G. Senjanovic and Y. Zhang, Phys. Rev. D 85 (2012) 055018 [arXiv:1108.4416 [hep-ph]]. 
[7] A. Arhrib, R. Benbrik, M. Chabab, G. Moultaka and L. Rahili, JHEP 1204 (2012) 136 [arXiv:1112.5453 [hep-ph]].

[8] S. Kanemura and K. Yagyu, Phys. Rev. D 85 (2012) 115009 [arXiv:1201.6287 [hep-ph]].

[9] A. G. Akeroyd and S. Moretti, arXiv:1206.0535 [hep-ph].

[10] M. Carena, I. Low and C. E. M. Wagner, arXiv:1206.1082 [hep-ph]. W. -F. Chang, J. N. Ng and J. M. S. Wu, arXiv:1206.5047 [hep-ph]. C. -W. Chiang and K. Yagyu, arXiv:1207.1065 [hep-ph]. H. An, T. Liu and L. -T. Wang, arXiv:1207.2473 [hep-ph]. A. Alves, A. G. Dias, E. R. Barreto, C. A. d. S. Pires, F. S. Queiroz and P. S. R. da Silva, arXiv:1207.3699 [hep-ph]. A. Joglekar, P. Schwaller and C. E. M. Wagner, arXiv:1207.4235 [hep-ph]. N. Arkani-Hamed, K. Blum, R. T. D'Agnolo and J. Fan, arXiv:1207.4482 [hep-ph]. L. G. Almeida, E. Bertuzzo, P. A. N. Machado and R. Z. Funchal, arXiv:1207.5254 [hep-ph]. A. Delgado, G. Nardini and M. Quiros, arXiv:1207.6596 [hep-ph]. J. Kearney, A. Pierce and N. Weiner, arXiv:1207.7062 [hep-ph]. I. Dorsner, S. Fajfer, A. Greljo and J. F. Kamenik, arXiv:1208.1266 [hep-ph]. K. Schmidt-Hoberg and F. Staub, arXiv:1208.1683 [hep-ph]. H. Davoudiasl, H. -S. Lee and W. J. Marciano, arXiv:1208.2973 [hep-ph]. K. J. Bae, T. H. Jung and H. D. Kim, arXiv:1208.3748 [hep-ph]. Y. Cai, W. Chao and S. Yang, arXiv:1208.3949 [hep-ph]. L. Wang and X. -F. Han, arXiv:1209.0376 [hep-ph].

[11] P. Achard et al. [L3 Collaboration], Phys. Lett. B 576 (2003) 18 [hep-ex/0309076].

[12] D. Acosta et al. [CDF Collaboration], Phys. Rev. Lett. 93, 221802 (2004) [hep-ex/0406073]; V. M. Abazov et al. [D0 Collaboration], Phys. Rev. Lett. 93 (2004) 141801 [hep-ex/0404015]. V. M. Abazov et al. [D0 Collaboration], Phys. Rev. Lett. 108, 021801 (2012) [arXiv:1106.4250 [hep-ex]].

[13] For a detail, see, e.g., E. J. Chun and P. Sharma, JHEP 1208 (2012) 162 [arXiv:1206.6278 [hep-ph]].

[14] P. Fileviez Perez, T. Han, G. -y. Huang, T. Li and K. Wang, Phys. Rev. D 78 (2008) 015018 [arXiv:0805.3536 [hep-ph]].

[15] C. -W. Chiang, T. Nomura and K. Tsumura, Phys. Rev. D 85, 095023 (2012) [arXiv:1202.2014 [hep-ph]].

[16] I. Gogoladze, N. Okada and Q. Shafi, Phys. Rev. D 78 (2008) 085005 [arXiv:0802.3257 [hep-ph]].

[17] A. Arhrib, R. Benbrik, M. Chabab, G. Moultaka, M. C. Peyranere, L. Rahili and J. Ramadan, Phys. Rev. D 84 (2011) 095005 [arXiv:1105.1925 [hep-ph]].

[18] M. A. Schmidt, Phys. Rev. D 76 (2007) 073010 [Erratum-ibid. D 85 (2012) 099903] [arXiv:0705.3841 [hep-ph]]; W. Chao and H. Zhang, Phys. Rev. D 75 (2007) 033003 [hep-ph/0611323].

[19] G. Isidori, G. Ridolfi and A. Strumia, Nucl. Phys. B 609 (2001) 387 [hep-ph/0104016].

[20] L. Lavoura and L. -F. Li, Phys. Rev. D 49 (1994) 1409 [hep-ph/9309262].

[21] M. Baak, M. Goebel, J. Haller, A. Hoecker, D. Kennedy, R. Kogler, K. Moenig and M. Schott et al., arXiv:1209.2716 [hep-ph].

[22] For a review and more references, see, A. Djouadi, Phys. Rept. 459 (2008) 1 [hep-ph/0503173]. 